In der Rubrik „Literatur kompakt" werden die wichtigsten Originalarbeiten aus der internationalen Fachliteratur referiert.

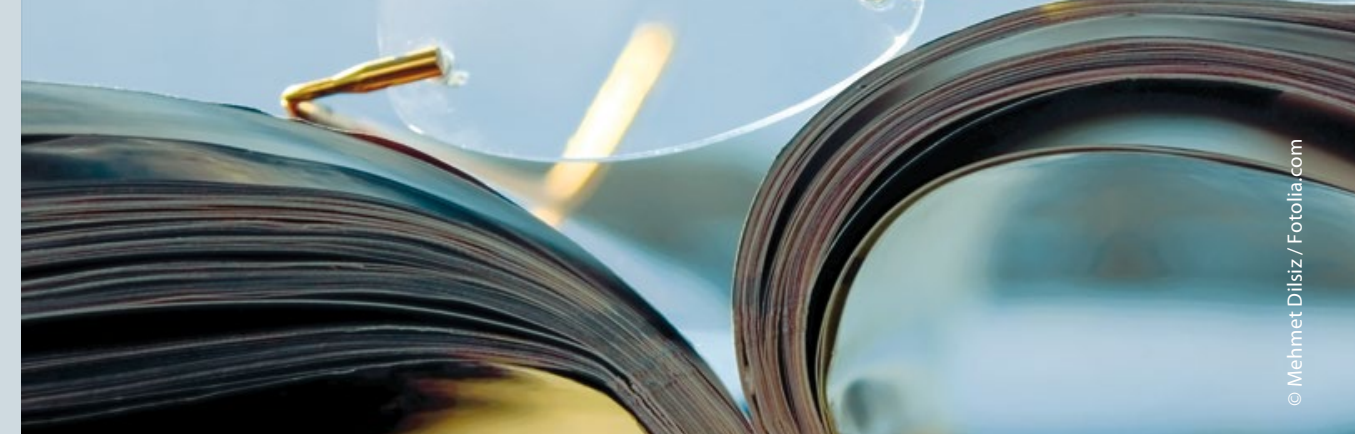

\section{Frequenz der Migräneattacken durch Akupunktur deutlich gesenkt}

Akupunktur ist für viele Migränepatienten eine Alternative zu Medikamenten. Die Erfahrungen auch in der Langzeitprophylaxe sind gut, eine große Scheinakupunktur- und Wartelisten-kontrollierte Studie liefert wissenschaftliche Belege für die Effektivität.

巨in großer Anteil der MigränepatienEten benötigt eine Prophylaxe. Pharmakotherapeutisch stehen unter anderem Topiramat, Metoprolol oder Propranolol zur Verfügung; limitiert wird deren Einsatz allerdings durch Nebenwirkungen wie Gewichtszunahme, Fatigue oder Schlafstörungen. Insbesondere Migränepatienten mit Medikamentenunverträglichkeit wählen als Alternative häufig die Akupunktur - sowohl therapeutisch als auch prophylaktisch. Die Wirksamkeit ist in kleineren Studien belegt. In einer multizentrischen Studie sollte speziell der Prophylaxeaspekt der Akupunktur gezeigt werden.

Rekrutiert wurden 249 erwachsene Patienten mit Migräne ohne Aura, die ambulant an drei Zentren für traditionelle chinesische Medizin in China betreut wurden. Randomisiert erhielten sie über vier Wochen an jeweils fünf Tagen entweder eine echte Akupunktur oder eine Scheinakupunktur, eine dritte Gruppe wurde in eine Warteliste eingetragen. Primäres Zielkriterium war die Veränderung der per Tagebuch aufgezeichneten Attackenfrequenz zwischen

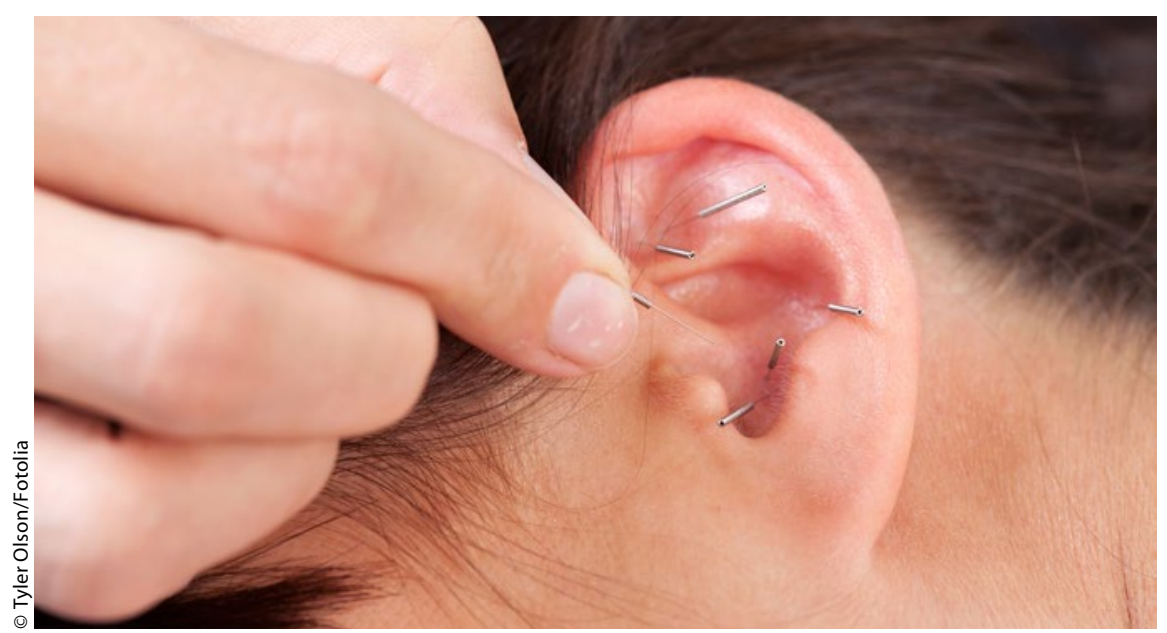

Akupunktur kann Migränepatienten prophylaktisch helfen.

Studienbeginn und Woche 16 der Behandlung.

Bei zu Studienbeginn vergleichbarer Attackenfrequenz bestanden in Woche 16 signifikante $(\mathrm{p}<0,001)$ Differenzen zwischen den drei Gruppen. Die durchschnittliche Kopfschmerzhäufigkeit pro Monat fiel bei den Patienten in der Akupunkturgruppe um 3,2 (Standardabweichung [SD] 2,1), in der Scheinakupunkturgruppe um 2,1 (SD 2,5) und in der Wartelistengruppe um 1,4 (SD 2,5), entsprechend einer Differenz von 1,1 Ereignissen pro Monat zwischen den Gruppe mit echter vs. Scheinakupunktur (95\%-Konfindenzintervall [KI] 0,4-1,9; $p=0,002)$ bzw. einer Differenz von 1,8 Ereignissen zwischen den Gruppen mit echter Akupunktur vs. Wartelistengruppe $(95 \%$-KI $1,1-2,5 ; \mathrm{p}<0,001)$. Die Unterschiede zwischen Scheinakupunktur vs. Warteliste waren nicht signifikant (Differenz 0,7 Attacken, 95\%-KI -0,1 bis $1,4 ; p=0,07)$. Auch die Schmerzintensität ging unter der Verumintervention deutlich zurück.

Fünf Patienten der Verumakupunkturgruppe und zwei Patienten der Scheinakupunkturgruppe gaben leichte Nebenwirkungen an, davon drei VerumPatienten ein Kribbeln der Kopf-Akupunkturpunkte während der Nadelung.

Fazit: Eine Akupunktur reduziert bei Migränepatienten ohne Aura die Attackenfrequenz pro Monat signifikant im Vergleich zu einer Scheinakupunktur. Auch die Intensität der Kopfschmerzen wurde geringer. Dr. Barbara Kreutzkamp

Zhao $L$ et al. The long-term effect of acupuncture for migraine prophylaxis. A randomized clinical trial. JAMA Intern Med 2017; 177(4):50815 